\title{
Calibração regional e local da equação de Hargreaves para estimativa da evapotranspiração de referência ${ }^{1}$
}

\author{
Regional and local calibration of Hargreaves equation for estimating reference \\ evapotranspiration
}

\author{
Diego Simões Fernandes ${ }^{2}$, Alexandre Bryan Heinemann ${ }^{2 *}$, Rosidalva Lopes Feitosa Paz e André de Oliveira \\ Amorim $^{3}$
}

\begin{abstract}
Resumo - A equação de Penman-Monteith FAO-56 $\left(\mathrm{ETo}_{\mathrm{PM}}\right)$ tem sido recomendada pela FAO, Organização para a Alimentação e Agricultura das Nações Unidas (ONU), como padrão para estimar a evapotranspiração de referência (ETo). Essa equação requer muitas variáveis que não estão disponíveis na maioria das estações meteorológicas no Brasil central. Por outro lado, a equação de Hargreaves é considerada simples e demanda somente dados de temperatura máxima e mínima para estimar a ETo. Entretanto, essa equação requer um ajuste local. Esse estudo analisa a possibilidade de utilizar a equação de Hargreaves ajustada para estimar a ETo no estado de Goiás. Para isso, os parâmetros empíricos, HC (coeficiente empírico de Hargreaves) e HE (expoente empírico de Hargreaves), da equação de Hargreaves foram ajustados considerando dois processos, ajuste local (HGR - Hargreaves ajuste local) e ajuste regional (HGL - Hargreaves ajuste regional). Para o HGL, os parâmetros empíricos foram ajustados para cada estação meteorológica. Já, para o HGR, os parâmetros empíricos foram ajustados considerando conjuntamente os dados de todas as estações meteorológicas. A equação de Hargreaves ajustada para ambos os processos, local e regional, apresentou valores de ERQM de 17,95 e 21,93\%, respectivamente, considerando o conjunto total de dados climáticos. A equação de Hargreaves ajustada localmente ou regionalmente é uma opção para estimar os valores diários de ETo no Estado de Goiás em locais em que a disponibilidade de dados climáticos é limitada.
\end{abstract}

Palavras-chave - Temperatura atmosférica. Evapotranspiração. Calibração.

\begin{abstract}
The FAO-56 Penman-Monteith equation $\left(\mathrm{ETo}_{\mathrm{PM}}\right)$ has been recommended by the Food and Agriculture Organization (FAO) of the United Nations as the standard equation for estimating reference evapotranspiration (ETo). The FAO-56 PM equation requires numerous weather data that are not available in most of the stations of Brazil central. On the other hand, the Hargreaves equation is a more simple equation for estimating ETo, and demands only maximum and minimum temperature data. However, this equation requires local calibration. This paper examines the potential of using the Hargreaves equation adjusted to estimate the ETo in Goiás State. For this, the Hargreaves empiric parameters, HC (empirical Hargreaves coefficient) and HE (empirical Hargreaves exponent), were adjusted considering two procedures, local adjustment (HGL - Hargreaves local adjustment) and regional adjustment (HGR - Hargreaves regional adjustment). For HGL, the adjustment of empiric parameters was done for each weather station. For HGR, the adjustment of empiric parameters was done considering the data set of all weather stations. The Hargreaves equation adjusted by both processes, local and regional, showed values of 17.95 and $21.93 \%$ for ERQM respectively, considering the full range of climatic data. The Hargreaves equation adjusted by both processes is an option to estimate the daily values of ETo in Goiás State where there are available data limitation.
\end{abstract}

Key words - Atmospheric Temperature. Evapotranspiration. Calibration.

\footnotetext{
* Autor para correspondência

${ }^{1}$ Recebido para publicação em 14/10/2010; aprovado em 06/09/2011

Pesquisa desenvolvida com recursos da Financiadora de Estudos e Projetos (FINEP)

${ }^{2}$ EMBRAPA Arroz e Feijão, Caixa Postal 179, Santo Antônio de Goiás-GO, Brasil, 75375-000, diegosifer@cnpaf.embrapa.br, alexbh@cnpaf.embrapa.br ${ }_{3}^{3}$ Sistema de Meteorologia e Hidrologia do Estado de Goiás (SIMEHGO)/SECTEC,Goiânia-GO, Brasil, 74088-900, dalvapaz@yahoo.com.br, amorim_go@yahoo.com.br
} 


\section{Introdução}

A necessidade de estimar a evapotranspiração de referência (ETo) com acurácia e por meio de um método simples vem sendo estudado por vários autores (ALLEN, 2003; FOOLADMAND et al., 2008; GAVILÁN et al., 2006; HARGREAVES; TRAJKOVIC, 2007). A razão é que o manejo da água e a produtividade das culturas podem ser melhorados em função da confiabilidade dos métodos utilizados para estimar a evapotranspiração das culturas. Além disso, compreender a ETo é essencial para um planejamento econômico do uso de recursos hídricos.

Vários métodos empíricos para a estimativa de ETo foram desenvolvidos para diferentes localidades e metodologias. Embora muitas equações tenham sido propostas para estimar a ETo, não há um consenso único para uma dada equação em um determinado clima. Assim, essas equações requerem uma calibração local (DEHGHANISANIJ et al., 2004). Um método utilizado universalmente para quantificar a ETo diária é o de Penman-Monteith FAO-56 (ALLEN et al., 1998). O principal problema desse método padrão é que requer medidas acuradas de temperatura do ar, umidade relativa do ar, radiação solar e velocidade do vento (PEREIRA et al., 2002; POPOVA et al., 2006). Infelizmente, há um número limitado de estações meteorológicas nos quais estas variáveis são medidas de modo eficiente, principalmente na região central do Brasil. Portanto, a busca por métodos alternativos que demandem menos variáveis meteorológicas para estimar a ETo tem sido uma solução viável para contornar esse problema. Um método alternativo que vem sendo utilizado por vários estudos (FOOLADMAND et al., 2008; GAVILÁN et al., 2006; TRAJKOVIC, 2007) é a equação de Hargreaves (HARGREAVES; SAMANI, 1985). Embora esta equação seja utilizada para estimar a ETo para períodos semanais ou maiores, existem estudos que ilustram que essa equação ajustada pode estimar com acurácia a ETo diária (ALLEN, 2003; FOOLADMAND et al., 2008; HARGREAVES; TRAJKOVIC, 2007). Essa equação estima a ETo baseada somente nos dados de temperatura do ar. Entretanto, requer uma calibração local para um desempenho aceitável (GAVILÁN et al., 2006). Essa calibração deve ser realizada por meio dos ajustes dos coeficientes da equação de Hargreaves. Isso porque calibrações utilizando apenas regressão linear superestimaram os valores da ETo quando comparadas aos valores obtidos por Penman-Monteith FAO-56 (ALLEN et al., 1994; TRAJKOVIC, 2005, 2007). Assim, os objetivos desse estudo foram calibrar a equação de Hargreaves para estimar a ETo diária por meio dos coeficientes empíricos de maneira local e regional, e analisar o desempenho desses ajustes para as diferentes épocas do ano no estado de Goiás.

\section{Material e métodos}

A região de estudo é o estado de Goiás, localizado entre os meridianos $45^{\circ}$ e $54^{\circ} \mathrm{O}$ e os paralelos $12^{\circ}$ e $20^{\circ} \mathrm{S}$, ocupando uma área aproximada de $340.000 \mathrm{~km}^{2}$. O clima é considerado tropical semi-úmido, com duas estações bem definidas, chuvosa (outubro - março) e seca (abril - setembro). As localizações geográficas das estações meteorológicas utilizadas nesse estudo estão ilustradas na Figura 1. Essas estações pertencem à rede de observação do Sistema de Meteorologia e Hidrologia do Estado de Goiás (SIMEHGO). Os municípios nos quais as estações estão localizadas, suas respectivas latitude, longitude, altitude, período e número de anos utilizados nesse estudo estão descritos na Tabela 1 .

Para cada estação meteorológica utilizou-se as variáveis climáticas, temperatura do ar máxima e mínima, umidade relativa máxima e mínima, velocidade do vento e radiaçãosolarglobal.Aqualidade dos dados meteorológicos provenientes das estações foram verificadas utilizando-se a metodologia descrita por Heinemann et al. (2007).

A partir destas variáveis foi estimada a evapotranspiração de referência (ETo) por meio do método de Penman-Montheith parametrizado no boletim 56 da FAO (eq. 1), visando calibrar os coeficientes da equação de Hargreaves, conforme metodologia proposta por Gavilán et al. (2006) e Nandagiri e Kovoor (2006).

$$
\mathrm{ETo}_{\mathrm{PM}}=\frac{0,408 \Delta\left(\mathrm{R}_{\mathrm{n}}-\mathrm{G}\right)+\gamma \frac{900}{\mathrm{~T}_{\text {med }}+273,16} \mathrm{U}_{2} \cdot\left(\mathrm{e}_{\mathrm{s}}-\mathrm{e}_{\mathrm{a}}\right)}{\Delta+\gamma\left(1+0,34 \mathrm{U}_{2}\right)}
$$

onde,

$\mathrm{ETo}_{\mathrm{PM}} \mathrm{ETo}_{\mathrm{PM}}$ - evapotranspiração de referência obtida pelo método de Penman-Monteith $\left(\mathrm{mmdia}^{-1}\right)$;

$\mathrm{R}_{\mathrm{n}}$ - saldo de radiação $\left(\mathrm{MJm}^{-2} \mathrm{dia}^{-1}\right)$;

$\mathrm{G}$ - densidade do fluxo de calor no solo, a qual é considerada zero para espaços de tempo de 24 horas $\left(\mathrm{MJ} \mathrm{m}^{-2} \mathrm{dia}^{-1}\right)$;

$\Delta$ - inclinação da curva de saturação do vapor de água $\left(\mathrm{kPa}{ }^{\circ} \mathrm{C}^{-1}\right)$;

$\mathrm{U}_{2}$ - velocidade do vento (média diária) a 2 metros acima da superfície do solo $\left(\mathrm{m} \mathrm{s}^{-1}\right)$;

$\mathrm{T}_{\text {med }}$ - temperatura média do ar a 2 metros acima da superfície do solo $\left({ }^{\circ} \mathrm{C}\right)$;

$\mathrm{e}_{\mathrm{s}}$ - a pressão de saturação do vapor $(\mathrm{kPa})$;

$\mathrm{e}_{\mathrm{a}}$ - pressão atual do vapor $(\mathrm{kPa})$;

$\mathrm{y}$ - fator psicrométrico $\left(\mathrm{kPa}{ }^{\circ} \mathrm{C}^{-1}\right)$.

A equação de Hargreaves, denominada aqui como $\mathrm{ETo}_{\mathrm{HG}}$, é descrita a seguir (eq. 2): 
Figura 1 - Mapa com a localização geográfica das estações meteorológicas utilizadas no estudo. Números representam as IDs das estações meteorológicas

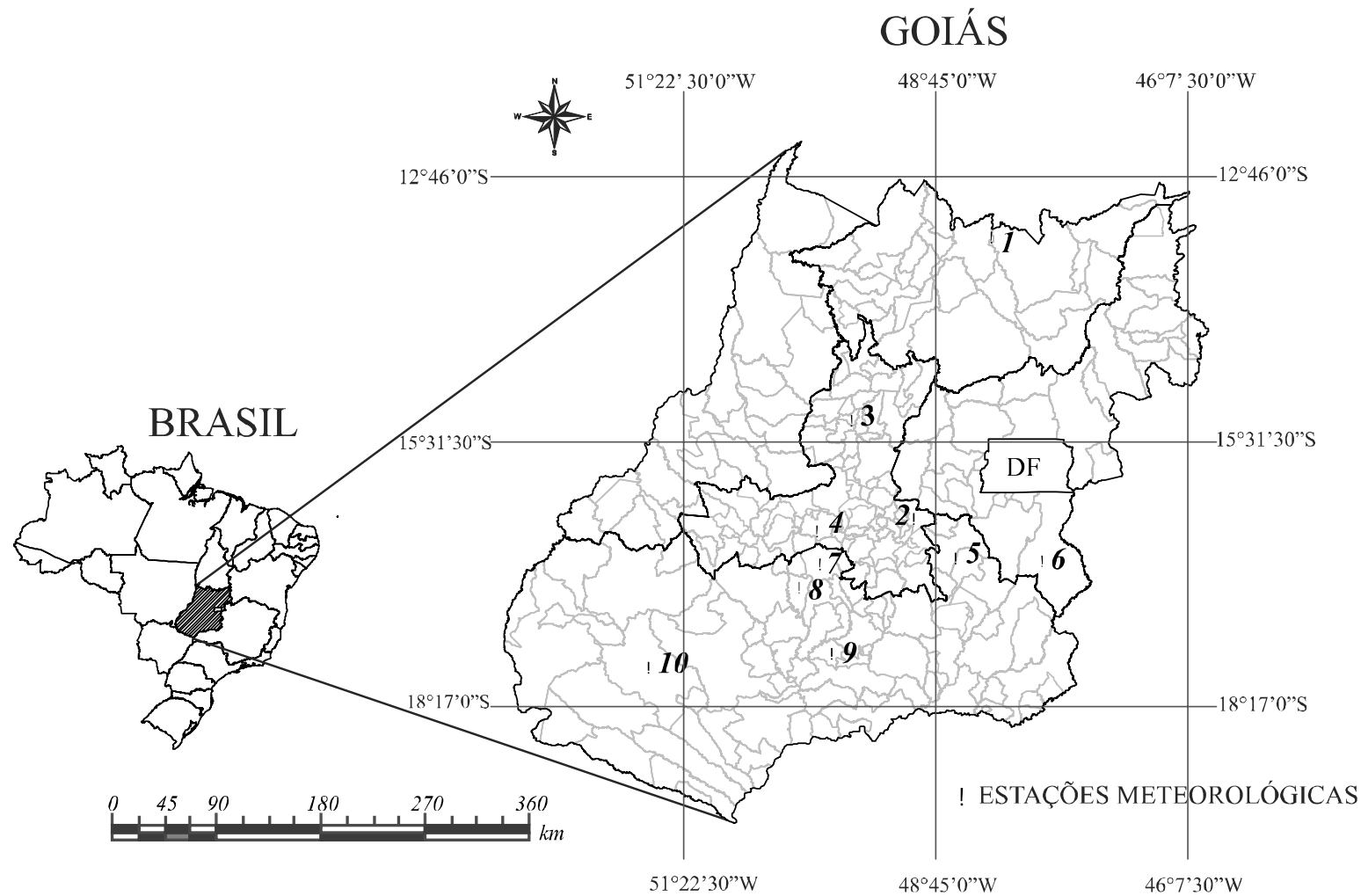

Tabela 1 - Localização geográfica, altitude, período e número de anos utilizados das estações meteorológicas avaliadas nesse estudo

\begin{tabular}{lcccccc}
\hline ID & Estações & Lat $\left(^{\circ}\right)$ & Lon $\left(^{\circ}\right)$ & Alt $(\mathrm{m})$ & Período & $\mathrm{N}^{\circ}$ de anos \\
\hline 01 & Minaçu & 13,04 & 48,05 & 343 & $2004-2009$ & 6 \\
02 & Ceres & 14,33 & 48,95 & 739 & $2002-2007$ & 6 \\
03 & Anápolis & 15,30 & 49,60 & 1136 & $2005-2008$ & 4 \\
04 & Anicuns & 16,46 & 49,96 & 692 & $2006-2009$ & 4 \\
05 & Vianópolis & 16,74 & 48,52 & 1110 & $2004-2007$ & 4 \\
06 & Cristalina & 16,77 & 47,61 & 1189 & $2005-2008$ & 4 \\
07 & Palmeiras de Goiás & 16,80 & 49,93 & 596 & $2000-2005$ & 6 \\
08 & Jandaia & 17,05 & 50,15 & 637 & $2005-2008$ & 4 \\
09 & Vicentinópolis & 17,74 & 49,81 & 648 & $2000-2005$ & 6 \\
10 & Jataí & 17,88 & 51,71 & 696 & $2004-2009$ & 6 \\
\hline
\end{tabular}

$\mathrm{ETo}_{\text {HG }}=0,408 \mathrm{HCR}_{\mathrm{a}}\left(\mathrm{T}_{\max }-\mathrm{T}_{\min }\right)\left(\frac{\left.\mathrm{T}_{\max }+\mathrm{T}_{\min }+17,8\right)}{2}\right.$

onde,

$\mathrm{ETo}_{\mathrm{HG}}$ - evapotranspiração de referência obtida pelo método de Hargreaves $\left(\mathrm{mm} \mathrm{dia}^{-1}\right)$;
$\mathrm{T}_{\max }$ - temperatura máxima diária do ar $\left({ }^{\circ} \mathrm{C}\right)$;

$\mathrm{T}_{\min }$ - temperatura mínima diária do ar $\left({ }^{\circ} \mathrm{C}\right)$;

$\mathrm{R}_{\mathrm{a}}$ - radiação extra terrestre $\left(\mathrm{MJm}^{-2} \mathrm{dia}^{-1}\right)$;

HC - coeficiente empírico da equação de Hargreaves, e;

HE - expoente empírico da equação de Hargreaves. 
Nesse estudo utilizou-se dois procedimentos para calibrar a equação $\mathrm{ETo}_{\mathrm{HG}}$, denominados local (HGL) e regional (HGR). O procedimento local foi realizado calibrando-se os parâmetros empíricos HC e HE para as condições climáticas de cada uma das estações meteorológicas descritas na Tabela 1. Já, para o procedimento regional, os coeficientes empíricos foram calibrados considerando conjuntamente os dados históricos de todas as estações meteorológicas. Para a calibração dos parâmetros empíricos de Hargreaves, em ambos os procedimentos, local e regional, utilizou-se somente os anos impares e para a validação, somente os anos pares. Essa metodologia reduz o efeito bias que pode ocorrer de um ano para outro.

A determinação dos parâmetros empíricos de Hargreaves, tanto para a calibração local como também regional, foram obtidas em função dos valores estimados de ETo, pela equação $\mathrm{ETo}_{\mathrm{PM}}$, utilizando-se o método de iteração para resolver a equação não linear (eq. 2). A função utilizada foi a nls (non linear least squares), do pacote MASS, do programa estatístico R (R Development Core Team, 2010).

Para quantificar o desempenho da equação $\mathrm{ETo}_{\mathrm{HG}}$ ajustada para o processo local e regional, utilizou-se os seguintes critérios estatísticos: coeficiente de determinação $\left(\mathrm{R}^{2}\right.$ ), o erro relativo do quadrado médio (ERQM, eq. 3),o erro médio absoluto (EMA, eq. 4) e a eficiência do modelo (EF, eq. 5).

$\mathrm{ERQM}=\frac{\left[\frac{\sum_{\mathrm{i}=1}^{\mathrm{N}}\left(\mathrm{ETo}_{\mathrm{PM}_{\mathrm{i}}}-\mathrm{ETo}_{\mathrm{HG}_{\mathrm{i}}}\right)^{2}}{\mathrm{~N}-1}\right]^{0,5}}{\bar{M}} \cdot 100$

onde,

ERQM - erro relativo do quadrado médio (\%);

$\mathrm{ETo}_{\mathrm{PMi}}$ - evapotranspiração de referência estimada pelo método de Penman-Monteith ( $\mathrm{mm} \mathrm{dia}^{-1}$ ) para um respectivo dia de um determinado ano (i);

$\mathrm{ETo}_{\mathrm{HGi}}$ - evapotranspiração de referência estimada pelo método de $\mathrm{ETo}_{\mathrm{HG}}$ local ou regional $\left(\mathrm{mm} \mathrm{dia}^{-1}\right)$ para um respectivo dia de um determinado ano;

$\mathrm{N}$ - número total de dias;

M - a média dos valores de ETo estimados pela equação de $\mathrm{ETo}_{\mathrm{PM}}$.

$$
\begin{aligned}
& \mathrm{EMA}=\frac{1}{\mathrm{~N}} \sum_{\mathrm{i}=1}^{\mathrm{N}}\left|\mathrm{ETo}_{\mathrm{PM}_{\mathrm{i}}}-\mathrm{ETo}_{\mathrm{HG}_{\mathrm{i}}}\right| \\
& \mathrm{EF}=1-\frac{\sum_{1}^{\mathrm{N}}\left(\mathrm{ETO}_{\mathrm{PMi}}-\mathrm{ETO}_{\mathrm{HGi}}\right)^{2}}{\sum_{1}^{\mathrm{N}}\left(\mathrm{ETO}_{\mathrm{PMi}^{-}}-\overline{\mathrm{ETO}}{ }_{\mathrm{PMi}}\right)^{2}}
\end{aligned}
$$

onde,

EF - eficiência do modelo;

EMA - erro médio absoluto $\left(\mathrm{mm} \mathrm{dia}^{-1}\right)$;

$\mathrm{ETo}_{\mathrm{PMi}}$ - média dos valores diários estimados pela equação de $\mathrm{ETo}_{\mathrm{PM}}\left(\mathrm{mm} \mathrm{dia}{ }^{-1}\right)$.

Um modelo perfeito deve ter a EF igual a 1 . Um valor igual a 0 significa que o modelo não prediz melhor que a média aritmética dos valores observados, ou seja, o modelo não serve para explicar a variabilidade nos valores observados.

\section{Resultados e discussão}

Os ajustes dos parâmetros empíricos $\mathrm{HC}$ e HE da equação de Hargreaves obtidos na calibração pelos processos local (HGL) e regional (HGR), com seus respectivos erros padrões e intervalos de confiança estão apresentados na Tabela 2. Os valores do coeficiente empírico HC, para HGL, variaram de 0,00047 (Anicuns) a 0,00157 (Jataí). Já, na calibração do HGR, para o mesmo coeficiente, o valor obtido foi de 0,00141. Esse valor está próximo do valor encontrado para a estação de Minaçu (0,00144). Essa variação nos valores do coeficiente empírico HC também foi observada por Gavilán et al. (2006), que calibraram a equação de $\mathrm{ETo}_{\mathrm{HG}}$ para diferentes locais na Espanha e obtiveram valores entre 0,00209 e 0,0029.

Os valores do expoente empírico $\mathrm{HE}$, para HGL, variaram de 0,59 (Jatai) a 1,11 (Anicuns). Coincidentemente, para ambas as estações, Jatai e Anicuns, foram obtidos os valores extremos dos parâmetros empíricos HC e HE. Já, para o HGR, o valor encontrado foi de 0,68 . Também, este valor está próximo ao da estação Minaçu $(0,65)$. Para ambos os parâmetros empíricos, HC e HE, o nível de significância foi menor que 0,001 (TAB. 2).

Ambos os parâmetros empíricos, $\mathrm{HC}$ e $\mathrm{HE}$, para a calibração local, não apresentaram tendência com o aumento ou diminuição da latitude. Isso pode ser observado na Tabela 2, na qual as estações estão organizadas da menor para a maior latitude, demonstrado a viabilidade de se obter coeficientes empíricos regionais.

A Tabela 3 descreve os índices de desempenho para a calibração e validação dos processos local (HGL) e regional (HGR). Para a calibração local (anos impares), a EF variou de 0,45 (Vicentinópolis) a 0,70 (Vianópolis). Ambas as estações, também, apresentaram os menores e maiores valores de ERQM e $\mathrm{R}^{2}$, respectivamente. $\mathrm{O}$ EMA foi menor que $1 \mathrm{~mm} \mathrm{dia}{ }^{-1}$ para todas as estações 
meteorológicas. Já, para a validação (anos pares) local, a EF aumentou para 7 estações meteorológicas (Minaçu, Anápolis, Ceres, Anicuns, Palmeiras de Goiás, Jandaia e Jataí). O EMA, também, foi menor que $1 \mathrm{~mm} \mathrm{dia}^{-1}$. A calibração regional (HGR) apresentou uma EF de 0,48, um pouco maior que a da estação de Vicentinópolis $(0,45)$ e o EMA foi de $0,79 \mathrm{~mm} \mathrm{dia}^{-1}$. Já, para a validação HGR, a EF aumentou e os ERQM e EMA diminuíram. Tanto para a calibração, como para a validação local
(HRL) e regional (HGR), houve uma tendência em superestimar os valores de ETo em relação aos valores obtidos pelo método padrão $\mathrm{ETo}_{\mathrm{PM}}$. Essa tendência, também, foi observada por Gavilán et al. (2006), Mudrik et al. (2002) e Oliveira et al. (2001). O desempenho da aplicação dos parâmetros empíricos $\mathrm{HC}$ e $\mathrm{HE}$ obtidos por meio da calibração regional (HGR) para cada estação meteorológica é descrito na Tabela 4. A EF, o ERQM, o EMA e o $\mathrm{R}^{2}$ variaram de 0,20 a 0,$70 ; 15,36$ a $26,89 \%$;

Tabela 2 - Valores dos coeficientes empíricos HC e HE da equação de Hargreaves para os processos de calibração local (HGL) e regional (HGR)

\begin{tabular}{|c|c|c|c|c|c|c|c|c|c|}
\hline \multirow{2}{*}{ Processo } & \multirow{2}{*}{$\begin{array}{c}\text { ID } \\
\text { Estação }\end{array}$} & \multirow[t]{2}{*}{$\mathrm{HC}$} & \multirow{2}{*}{$\begin{array}{l}\text { Erro } \\
\text { Padrão }\end{array}$} & \multicolumn{2}{|c|}{$\begin{array}{c}\text { Intervalo de } \\
\text { Confiança }(\%)\end{array}$} & \multirow[t]{2}{*}{$\mathrm{HE}$} & \multirow{2}{*}{$\begin{array}{l}\text { Erro } \\
\text { Padrão }\end{array}$} & \multicolumn{2}{|c|}{$\begin{array}{c}\text { Intervalo de } \\
\text { Confiança (\%) }\end{array}$} \\
\hline & & & & 2,5 & 97,5 & & & 2,5 & 97,5 \\
\hline \multirow{10}{*}{ HGL } & 01 & $0,00144 *$ & 0,000065 & 0,00131 & 0,00156 & $0,65^{*}$ & 0,018 & 0,62 & 0,69 \\
\hline & 02 & $0,00111^{*}$ & 0,000046 & 0,00102 & 0,00120 & $0,75^{*}$ & 0,015 & 0,72 & 0,78 \\
\hline & 03 & $0,00076^{*}$ & 0,000054 & 0,00066 & 0,00087 & $1,00^{*}$ & 0,028 & 0,95 & 1,06 \\
\hline & 04 & $0,00047 *$ & 0,000034 & 0,00040 & 0,00054 & $1,11^{*}$ & 0,028 & 1,05 & 1,16 \\
\hline & 05 & $0,00109 *$ & 0,000057 & 0,00098 & 0,00120 & $0,76^{*}$ & 0,019 & 0,72 & 0,80 \\
\hline & 06 & $0,00084 *$ & 0,000060 & 0,00072 & 0,00096 & $0,96^{*}$ & 0,030 & 0,90 & 1,02 \\
\hline & 07 & $0,00094^{*}$ & 0,000055 & 0,00083 & 0,00104 & $0,76^{*}$ & 0,021 & 0,72 & 0,80 \\
\hline & 08 & $0,00097 *$ & 0,000064 & 0,00084 & 0,00109 & $0,88^{*}$ & 0,026 & 0,83 & 0,93 \\
\hline & 09 & $0,00074 *$ & 0,000058 & 0,00062 & 0,00085 & $0,96^{*}$ & 0,031 & 0,90 & 1,02 \\
\hline & 10 & $0,00157^{*}$ & 0,000060 & 0,00145 & 0,00168 & $0,59 *$ & 0,015 & 0,56 & 0,61 \\
\hline HGR & Todas & $0,00141 *$ & 0,000003 & 0,00135 & 0,00146 & $0,68^{*}$ & 0,008 & 0,66 & 0,69 \\
\hline
\end{tabular}

* nível de significância $<0,001$

Tabela 3 - Desempenho das estimativas de evapotranspiração para (a) a calibração dos processos local (HGL) e regional (HGR), (b) validação dos processos local (HGL) e regional (HGR)

\begin{tabular}{|c|c|c|c|c|c|c|c|c|c|}
\hline \multirow{2}{*}{ Processo } & \multirow{2}{*}{$\begin{array}{c}\text { ID } \\
\text { Estações }\end{array}$} & \multicolumn{4}{|c|}{ (a) } & \multicolumn{4}{|c|}{ (b) } \\
\hline & & *EF & ${ }^{\wedge} \mathrm{ERQM} \%$ & ${ }^{\&}$ EMA mm dia $^{-1}$ & $* * \mathrm{R}^{2}$ & $\overline{E F}$ & ERQM \% & EMA mm dia $^{-1}$ & $\mathrm{R}^{2}$ \\
\hline \multirow{10}{*}{ HGL } & 1 & 0,62 & 17,92 & 0,56 & 0,62 & 0,72 & 15,09 & 0,49 & 0,73 \\
\hline & 2 & 0,69 & 15,25 & 0,56 & 0,69 & 0,77 & 13,69 & 0,48 & 0,77 \\
\hline & 3 & 0,65 & 18,95 & 0,70 & 0,66 & 0,69 & 18,04 & 0,64 & 0,70 \\
\hline & 4 & 0,68 & 18,61 & 0,66 & 0,68 & 0,76 & 16,95 & 0,59 & 0,76 \\
\hline & 5 & 0,70 & 15,39 & 0,53 & 0,70 & 0,69 & 18,72 & 0,55 & 0,70 \\
\hline & 6 & 0,63 & 18,88 & 0,68 & 0,66 & 0,61 & 20,37 & 0,67 & 0,63 \\
\hline & 7 & 0,64 & 16,07 & 0,54 & 0,64 & 0,74 & 13,7 & 0,47 & 0,75 \\
\hline & 8 & 0,59 & 19,27 & 0,78 & 0,6 & 0,68 & 18,01 & 0,68 & 0,72 \\
\hline & 9 & 0,45 & 22,04 & 0,87 & 0,52 & 0,45 & 22,99 & 0,92 & 0,50 \\
\hline & 0 & 0,67 & 16,43 & 0,47 & 0,67 & 0,72 & 15,73 & 0,45 & 0,72 \\
\hline HGR & Todas & 0,48 & 22,17 & 0,79 & 0,49 & 0,54 & 21,72 & 0,75 & 0,55 \\
\hline
\end{tabular}

*EF - eficiência do modelo; ${ }^{\wedge} \mathrm{ERQM}$ - erro relativo do quadrado médio; ${ }^{\circledR} \mathrm{EMA}$ - erro médio absoluto; ** $\mathrm{R}^{2}$ - coeficiente de determinação 
0,56 a $1,00 \mathrm{~mm} \mathrm{~d}^{-1}$ e 0,42 a 0,73 , respectivamente. Como se pode observar, a utilização de valores dos parâmetros empíricos $\mathrm{HE}$ e $\mathrm{HC}$ obtidos pelo processo regional não teve o mesmo desempenho que os valores obtidos pelo processo local. Entretanto, para o processo HGR, os maiores valores para EMA e ERQM foram de $1 \mathrm{~mm} \mathrm{dia}^{-1}$ e $27 \%$, o que pode ser considerado razoável.

Tabela 4 - Desempenho da estimativa de evapotranspiração para o processo regional (HGR) aplicado para as diferentes estações

\begin{tabular}{ccccc}
\hline Processo & $\begin{array}{c}\text { ID } \\
\text { Estações }\end{array}$ & $\begin{array}{c}\text { ERQM } \\
(\%)\end{array}$ & $\begin{array}{c}\text { EMA } \\
\left(\mathrm{mm} \mathrm{dia}^{-1}\right)\end{array}$ & $\mathrm{R}^{2}$ \\
\hline \multirow{6}{*}{ HGR } & 1 & 17,39 & 0,57 & 0,67 \\
& 2 & 15,36 & 0,58 & 0,73 \\
& 3 & 26,89 & 1,00 & 0,58 \\
& 4 & 21,35 & 0,78 & 0,62 \\
& 6 & 17,28 & 0,56 & 0,71 \\
& 7 & 25,50 & 0,89 & 0,57 \\
& 8 & 23,19 & 0,93 & 0,69 \\
& 9 & 25,16 & 0,93 & 0,61 \\
& 10 & 20,88 & 0,67 & 0,42 \\
\hline
\end{tabular}

A Figura 2 ilustra os histogramas e densidades para as distribuições da ETo estimada pelos métodos de $\mathrm{ETo}_{\mathrm{PM}}$ e $\mathrm{ETo}_{\mathrm{HG}}$ para o processo local (HGL) e regional (HGR) considerando conjuntamente a série histórica de dados climáticos das 10 estações (FIG. 2a e b), os meses chuvosos (outubro a março) (FIG. 2c e d) e os meses secos (FIG. 2e e f). Por meio da Figura 2, pode-se observar que a ETo estimada por $\mathrm{ETo}_{\mathrm{PM}}$ e $\mathrm{ETo}_{\mathrm{HG}}$, tanto pelo processo local (HRL) e regional (HGR), apresentaram uma distribuição normal. Considerando conjuntamente os dados climáticos das 10 estações meteorológicas (FIG. 2 a e b) observou-se que ambos os processos, HGL (FIG. 2a) e HGR (FIG. 2b), superestimaram a ETo para valores entre 3,5 e 5,5 $\mathrm{mm} \mathrm{dia}^{-1}$. A diferença entre ambos os processos, HGL e HGR, para essa faixa de valores de ETo, é que HGL apresentou uma maior acurácia. Para valores menores que 3,5 e maiores que 5,5 $\mathrm{mm} \mathrm{dia}^{-1}$, a HGL mostrou basicamente a mesma tendência da equação de $\mathrm{ETo}_{\mathrm{PM}}$. Já o HGR subestimou os valores de ETo calculado pelo método padrão $\mathrm{ETo}_{\mathrm{PM}}$. Como pode ser observado na Tabela 5a, o ERQM e o EMA foram menores e a $\mathrm{EF}(0,67)$ maior para o processo HGL. Conforme ilustra a Figura 3 ( a e b), o $\mathrm{R}^{2}$ também foi maior para o HGL. Por meio dessas figuras, observa-se que a linha de regressão no processo HGL está mais próxima da linha 1:1 do que para o processo HGR. Isso confirma o fato do processo HGR superestimar a ETo mais que o HGL. Os valores de ETo mais freqüentes foram entre 4,0 e 4,5 mm $\mathrm{dia}^{-1}$. Considerando-se somente o período chuvoso (outubro a março), Figura 2c e d, a HGL e HGR subestimaram e superestimaram a ETo para valores menores e maiores que $4 \mathrm{~mm} \mathrm{dia}{ }^{-1}$, respectivamente. Nesse caso, a EF aumentou para ambos os processos (TAB. 5b). Já, o ERQM e o EMA diminuíram tanto para o HGL como para o HGR. Conforme ilustra a Figura 3 (c e d), o $\mathrm{R}^{2}$ aumentou para ambos os processos. Nesse período, os valores de ETo mais freqüentes foram entre 4,5 a 5,0 $\mathrm{mm} \mathrm{dia}{ }^{-1}$. Já, para o período seco (abril a setembro), Figura 2 (e e f), o HGL e HGR superestimaram para valores entre 3,0 mm dia ${ }^{-1}$ e 5,0 $\mathrm{mm} \mathrm{dia}^{-1}$ e subestimaram para valores maiores que 5,0 $\mathrm{mm} \mathrm{dia}^{-1}$. Para esse período, ambos os processos, HGL e HGR, apresentaram o pior desempenho (TAB. 5c). Conforme ilustra a Figura 3 (e e f), o $\mathrm{R}^{2}$ também foi o menor para ambos os processos. Nesse período os valores mais freqüentes de ETo foram entre 4,0 e 4,5 $\mathrm{mm} \mathrm{dia}^{-1}$.

Conforme descrito anteriormente, a equação de Penman-Monteith FAO-56 é recomendada como padrão para estimar a ETo de referência. Entretanto, o uso desse método é limitado devido à escassa disponibilidade de variáveis meteorológicas. Assim, a hipótese desse estudo baseia-se na utilização da equação de $\mathrm{ETo}_{\mathrm{HG}}$ ajustada

Tabela 5 - Desempenho das equações de Hargreaves ajustada localmente (HGL) e regionalmente (HGR) para (a) todo o conjunto de dados climáticos das 10 estações meteorológicas, (b) para os meses chuvosos (outubro a março) e (c) para os meses secos (abril a setembro)

\begin{tabular}{|c|c|c|c|c|c|c|c|c|c|}
\hline \multirow[b]{2}{*}{ Processo } & \multicolumn{3}{|c|}{-----------------(a)---------------- } & \multicolumn{3}{|c|}{-----------------(b)----------------- } & \multicolumn{3}{|c|}{-----------------(c)----------------- } \\
\hline & $\mathrm{EF}^{*}$ & $\begin{array}{c}\mathrm{ERQM}^{\wedge} \\
\%\end{array}$ & $\begin{array}{c}\mathrm{EMA}^{\&} \\
\mathrm{~mm} \mathrm{dia}{ }^{-1}\end{array}$ & $\mathrm{EF}$ & $\begin{array}{c}\text { ERQM } \\
\%\end{array}$ & $\begin{array}{c}\text { EMA } \\
\text { mm dia-1 }\end{array}$ & $\mathrm{EF}$ & $\begin{array}{c}\text { ERQM } \\
\%\end{array}$ & $\begin{array}{c}\text { EMA } \\
\text { mm dia }{ }^{-1}\end{array}$ \\
\hline Local (HGL) & 0,67 & 17,95 & 0,61 & 0,72 & 17,68 & 0,60 & 0,62 & 18,18 & 0,62 \\
\hline Regional (HGR) & 0,51 & 21,93 & 0,76 & 0,64 & 19,86 & 0,69 & 0,35 & 23,63 & 0,84 \\
\hline
\end{tabular}

${ }^{*} \mathrm{EF}$ - eficiência do modelo; ${ }^{\wedge}$ ERQM - erro relativo do quadrado médio; ${ }^{\circledR}$ EMA - erro médio absoluto 
Figura 2 - Densidade relativa e histograma de freqüência (branco - Hargreaves, cinza - Penman-Monteith) dos valores de ETo estimado por Hargreaves e Penman-Monteith pelo processo local e regional (a e b) considerando-se todos os meses da serie histórica de dados das 10 estações meteorológicas; (c e d) considerando-se somente os meses chuvosos (outubro a março); e (e e f) considerando-se somente os meses secos(abril a setembro)

Local
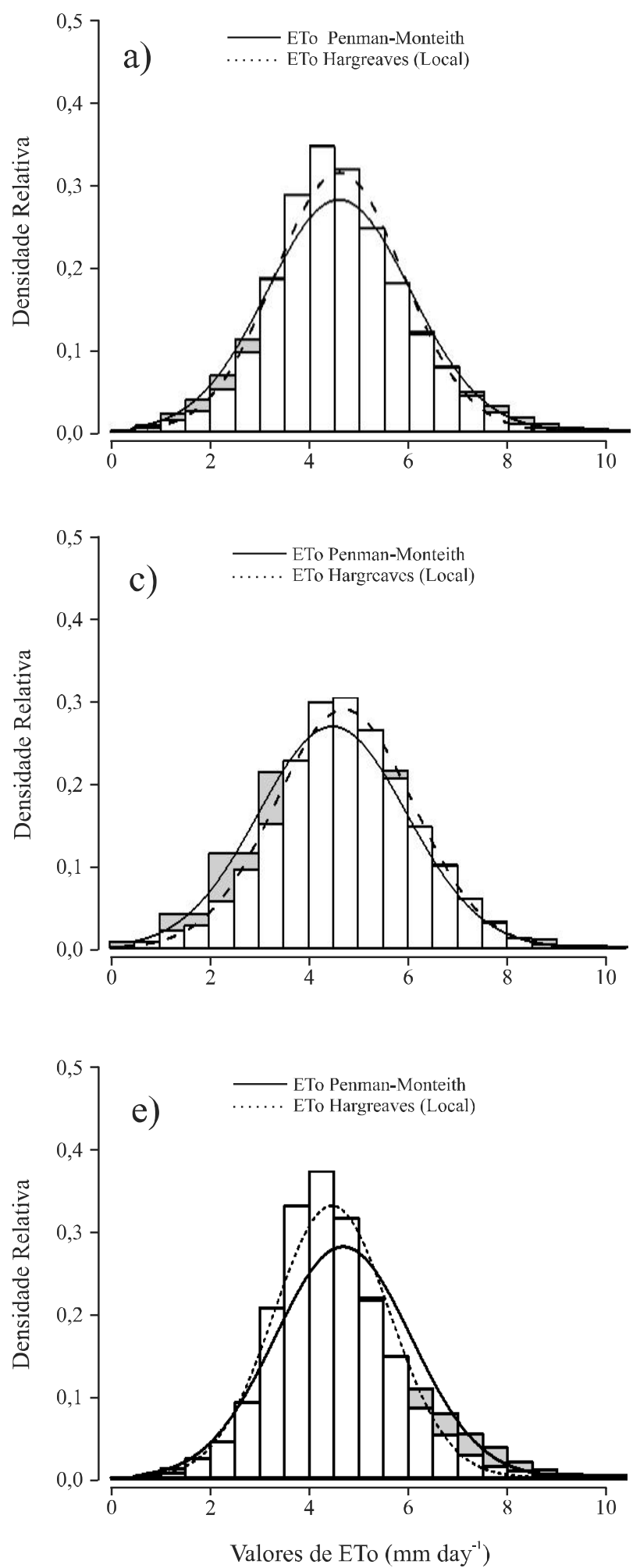

Regional
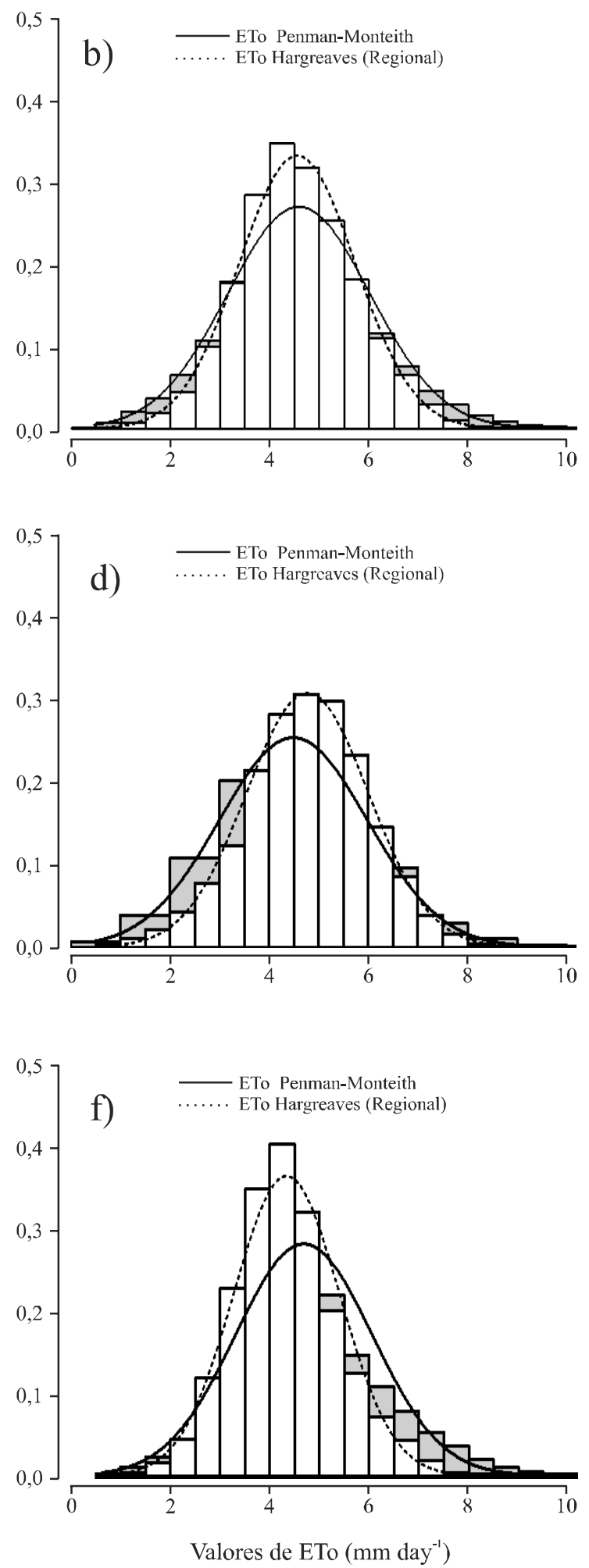
Figura 3 - Regressão entre a evapotranspiração de referência (ETo) estimada pela equação de Hargreaves e Penman-Monteith. (a e b) considerando-se todos os meses da serie histórica de dados das 10 estações meteorológicas; (c e d) considerando-se somente os meses chuvosos (outubro a março); e (e e f) considerando-se somente os meses secos (abril a setembro). Linha de regressão (tracejada) e linha 1:1 (contínua)

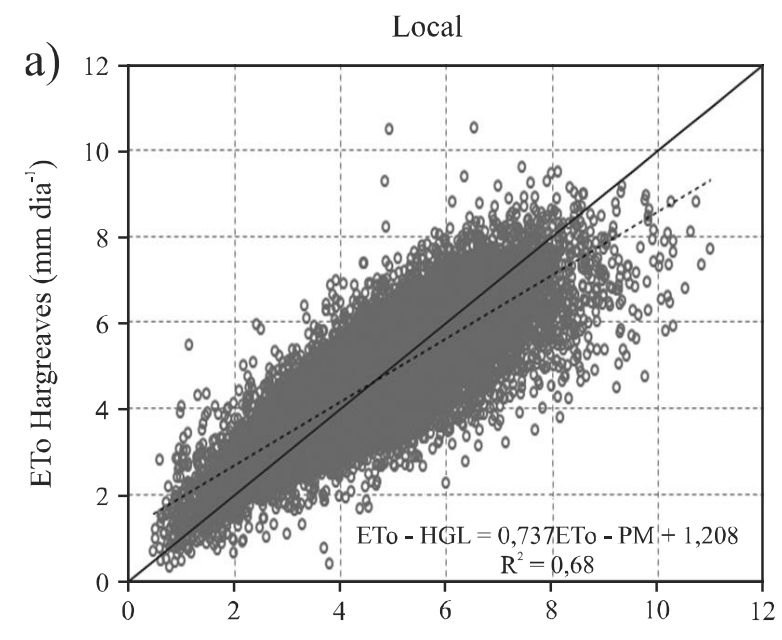

ETo Penman-Monteith $\left(\mathrm{mm} \mathrm{dia}^{-1}\right)$

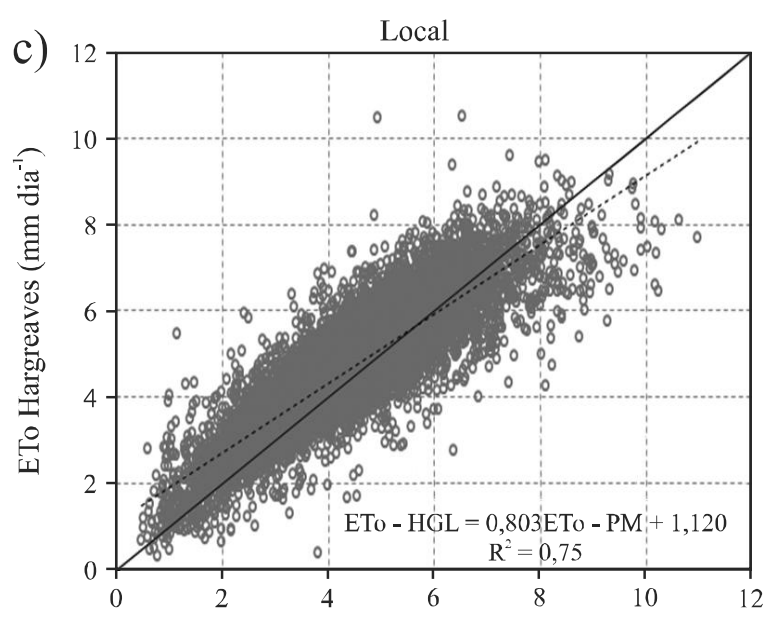

ETo Penman-Monteith (mm dia $\left.{ }^{-1}\right)$

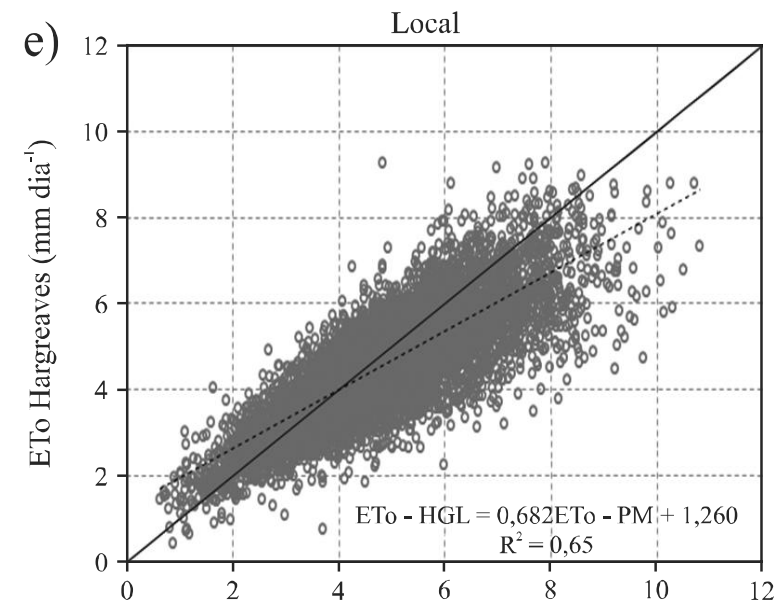

ETo Penman-Monteith ( $\left.\mathrm{mm} \mathrm{dia}^{-1}\right)$

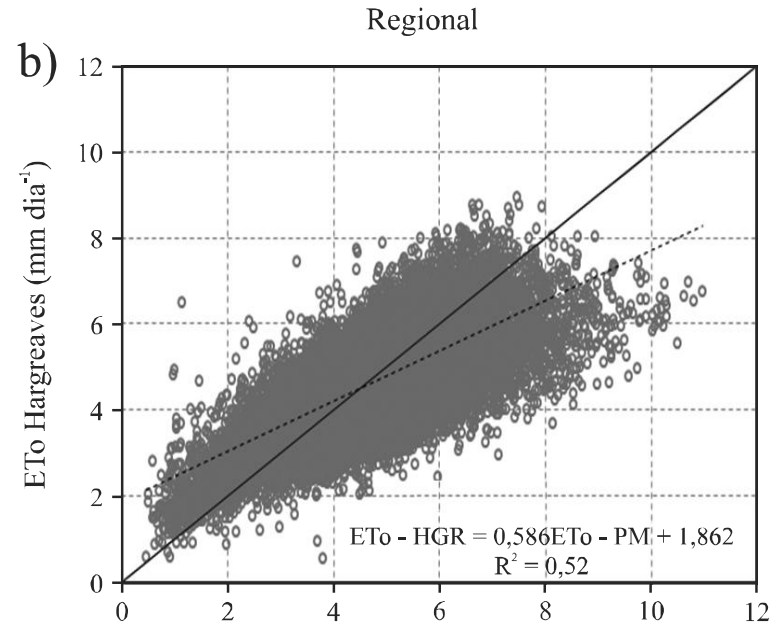

ETo Penman-Monteith (mm dia $\left.{ }^{-1}\right)$

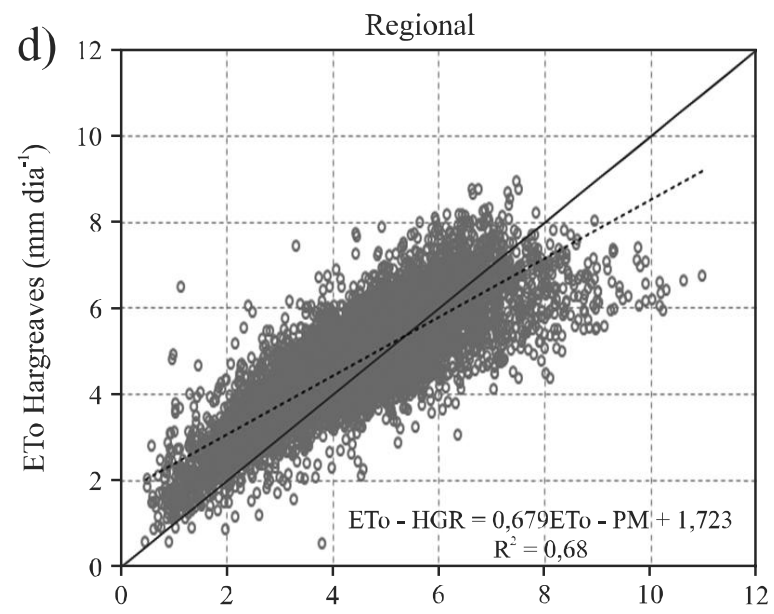

ETo Penman-Monteith ( $\left.\mathrm{mm} \mathrm{dia}{ }^{-1}\right)$

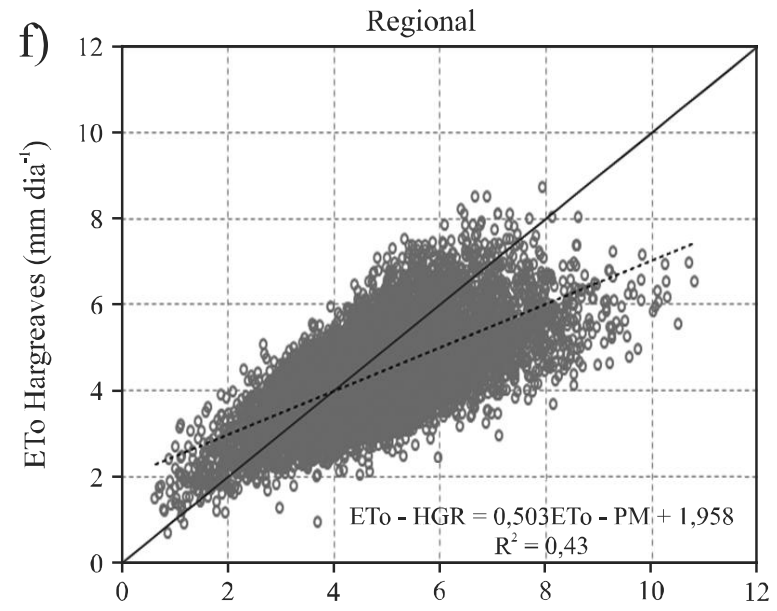

ETo Penman-Monteith ( $\left.m m \operatorname{dia}^{-1}\right)$ 
de maneira local e regional para estimar a ETo diária. Basicamente, para ambos os processos de ajuste (HGL e HGR), a equação de $\mathrm{ETo}_{\mathrm{HG}}$ superestimou os valores de ETo. Jabloun e Sahli (2008) também observaram que a equação de $\mathrm{ETo}_{\mathrm{HG}}$ superestima os valores de ETo para o interior da Tunísia, mas os mesmos não ajustaram os coeficientes empíricos da equação para a região de estudo. Entretanto, devidoà simplicidade daequação de Hargreaves ajustada localmente (HGL) e regionalmente (HGR) e seus respectivos desempenhos, existe a possibilidade de utilizar as mesmas para o estado de Goiás com a finalidade de estimar valores diários de ETo, em função da escassez de dados meteorológicos na região. Por meio da utilização da equação de $\mathrm{ETo}_{\mathrm{HG}}$ ajustada, há a possibilidade de se fazer um manejo da irrigação utilizando-se somente as variáveis meteorológicas temperaturas máximas e mínimas, as quais podem ser obtidas através de sensores economicamente acessíveis e robustos. Também, devido à existência de séries históricas de dados diários de temperatura máxima e mínima, possibilita a estimativa de séries temporais de ETo para um melhor planejamento dos recursos hídricos.

\section{Conclusões}

1. A equação de Hargreaves ajustada localmente e regionalmente superestimam os valores diários de ETo comparados com a equação de Penman-Monteith FAO-56;

2. A equação de Hargreaves ajustada por meio de seus coeficientes empíricos de maneira local ou regional é uma alternativa para estimar a ETo diária em locais em que a disponibilidade de dados climáticos é limitada.

\section{Agradecimentos}

Os autores agradecem ao MCT/FINEP pelo suporte financeiro dado a essa pesquisa e ao CNPq pela bolsa de desenvolvimento tecnológico e industrial do primeiro autor.

\section{Referências}

ALLEN, R. G. et al. An update for the definition of reference evapotranspiration.ICID Bulletin, v. 43, p. 1-34, 1994.

ALLEN, R. G. et al. Crop evapotranspiration: guidelines for computing crop water requirements. Rome: FAO, 1998. 370 p. (FAO. Irrigation and Drainage Paper, 56).

DEHGHANISANIJ, H.; YAMAMOTOA, T.; RASIAH, V. Assessment of evapotranspiration estimation models for use in semi-arid environments. Agricultural Water Management, v. 64, n. 02, p. 91-106, 2004.

FOOLADMAND, H. R.; ZANDILAK, H.; RAVANAN, M. H. Comparison of different types of Hargreaves equation for estimating monthly evapotranspiration in the south of Iran. Archives of Agronomy and Soil Science, v. 54, n. 03, p. 321-330, 2008.

GAVILÁN, P. et al. Regional calibration of Hargreaves equation for estimating reference ET in a semiarid environment. Agricultural Water Management, v. 81, n. 03, p. 257-281, 2006

HARGREAVES, G. H.; SAMANI, Z. A. Reference crop evapotranspiration from temperature. Applied Engineering in Agriculture, v. 01, n. 02, p. 96-99, 1985.

HARGREAVES, G. H.; ALLEN, R. G. History and evaluation of Hargreaves evapotranspiration equation. Journal of Irrigation and Drainage Engineering, v. 129, n. 53, p. 53-63, 2003.

HEINEMANN, A. B. et al. Características climáticas dos municípios de Santo Antônio de Goiás (GO), Porangatu (GO), Janaúba (MG), Sete Lagoas (MG), Parnaíba (PI) e Teresina (PI). Santo Antônio de Goiás: Embrapa Arroz e Feijão, 2007. 36 p. (Embrapa Arroz e Feijão. Documentos, 214).

JABLOUN, M.; SAHLI, A. Evaluation of FAO-56 methodology for estimating reference evapotranspiration using limited climatic data Application to Tunisia. Agricultural Water Management, v. 95, n. 6, p. 707-715, 2008.

MUDRIK, A. S. et al. Comparação da evapotranspiração de referencia (ETo), estimada pelos modelos teóricos de Penman-Monteith e Hargreaves-Samani, com uso do software SISDA. In: SIMPÓSIO BRASILEIRO DE PESQUISA EM CAFEICUlTURA IRRIGADA, 5., Araguari. Anais... Uberlândia: Universidade Federal de Uberlândia, 2002. p. 129-132.

NANDAGIRI, L.; KOVOOR, G. M. Performance evaluation of reference evapotranspiration equations across a range of Indian climates. Journal of Irrigation and Drainage Engineering, v. 132, n. 03, p. 238-249, 2006.

OLIVEIRA, L. F. C. et al. Estudo comparativo de modelos de estimativa da evapotranspiração de referência para algumas localidades no Estudo de Goiás e Distrito Federal. Pesquisa Agropecuária Tropical, v. 31, n. 02, p. 121-126, 2001.

PEREIRA, A. R. et al. Substantiation of the daily FAO56 reference evapotranspiration with data from automatic and conventional weather stations. Revista Brasileira de Agrometeorologia, v. 10, n. 02, p. 251-257, 2002.

POPOVA, Z.; KERCHEVA, M.; PEREIRA, L. S. Validation of the FAO methodology for computing ETo with limited data. Irrigation and Drainage, v. 55, p. 201-215, 2006.

R Development Core Team (2010). R: A language and environment for statistical computing, reference index version 2.12.1. R Foundation for Statistical Computing, Vienna, Austria. ISBN 3-900051-07-0, URL http://www.R-project.org>. 
TRAJKOVIC, S. Temperature-based approaches for estimating reference evapotranspiration. Journal of Irrigation and Drainage Engineering, v. 131, n. 04, p. 316-323, 2005.
TRAJKOVIC, S. Hargreaves versus Penman-Monteith under humid conditions. Journal of Irrigation and Drainage Engineering, v. 133, n. 01, p. 38-42, 2007. 